\title{
Transparency and amplification in a hybrid system of mechanical resonator and circuit QED
}

\author{
Hui Wang, ${ }^{1}$ Hui-Chen Sun, ${ }^{1}$ Jing Zhang, ${ }^{2,3}$ and Yu-xi Liu ${ }^{1,2}$ \\ ${ }^{1}$ Institute of Microelectronics, Tsinghua University, Beijing 100084, China \\ ${ }^{2}$ Tsinghua National Laboratory for Information Science and Technology (TNList), Tsinghua University, Beijing 100084, China \\ ${ }^{3}$ Department of Automation, Tsinghua University, Beijing 100084, China
}

(Dated: January 16, 2021)

\begin{abstract}
We theoretically study the transparency and amplification of a weak probe field applied to the cavity in hybrid systems formed by a driven superconducting circuit QED system and a mechanical resonator, or a driven optomechanical system and a superconducting qubit. We find that both the mechanical resonator and the superconducting qubit can result in the transparency to a weak probe field in such hybrid systems when a strong driving field is applied to the cavity. We also find that the weak probe field can be amplified in some parameter regimes. We further study the statistical properties of the output field via the degrees of second-order coherence. We find that the nonclassicality of the output field strongly depends on the system parameters. Our studies show that one can control single-photon transmission in the optomechanical system via a tunable artificial atom or in the circuit QED system via a mechanical resonator.
\end{abstract}

PACS numbers:

\section{INTRODUCTION}

It is well known that an incident light through a cavity without internal loss can be completely transmitted (or reflected) when it is resonant with (or far from resonance to) the mode of the cavity in the steady-state. However, this situation can be changed by impurities inside the cavity or unstable boundary condition of the cavity. When these impurities are considered as two-level atoms, a cavity quantum electrodynamics system can be formed. The unstable boundary condition can be described by an oscillating mirror at the one-end of the cavity, and then the cavity together with an oscillating mirror becomes an optomechanical system.

In optomechanical systems [1], when a strong driving field is applied to the cavity, there is an analogue of electromagnetically induced transparency (EIT) for the output at the frequency of the weak detecting field [2]. Such EIT phenomenon is equivalent to that in two coupled harmonic oscillators [3], which has been demonstrated in metamaterials [4]. The optomechanically induced EIT and slow light have been experimentally demonstrated [5-7]. However when an atomic ensemble is coupled to a cavity field in the optomechanical system, it was showed that two-level atomic ensemble can not only be used to enhance the photon-phonon coupling through the radiation pressure [8], but also be used to broaden the transparency windows [9]. We have also showed that the EIT in a three-level atomic ensemble, which is placed inside a cavity of the optomechanical system, can be significantly changed by the oscillating mirror [10].

With rapid progress in the research on superconducting qubits, scientists are now studying the quantum switch (e.g., Ref. [11]) by using the mechanical resonator for information transfer between different qubits, and also experimentalists started to demonstrate optomechanical effect in the microwave regime. For optomechanical systems in the microwave regime, the cavity is usually realized by a superconducting transmission line resonator, the mechanical resonator is realized by a suspended aluminum membrane [12], or a beam of conducting aluminium clamped on both ends [13],

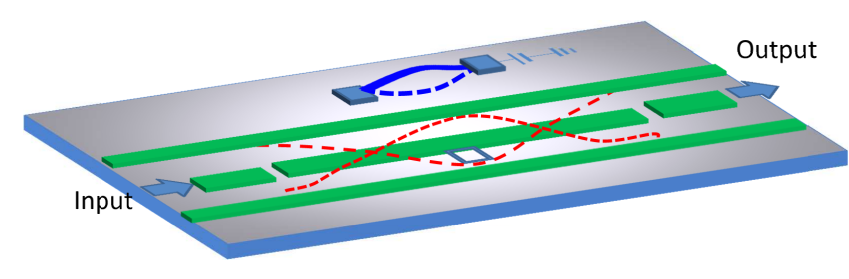

FIG. 1: (Color online) Schematic diagram for the circuit QED which is coupled to a nanomechanical resonator or an optomechanical system in microwave regime which is coupled to a superconducting qubit.

or a silicon nitride nano-structure [14]. Moreover, the circuit QED, which describes the interaction between a quantized microwave field and superconducting qubits (called as superconducting artificial atoms), is explored for superconducting quantum information processing [15, 16]. We have shown that the electromagnetically induced transparency and absorption can be tuned in circuit QED systems by virtue of the dressed three-level systems [17]. In contrast to the original proposal for EIT-induced photon blockade using atomic medium [18], the strong photon-qubit interaction in circuit QED systems makes the photon blockade become possible at the singleatom level, and also the photon blockade in the microwave regime has been experimentally demonstrated [19, 20]. Recent studies showed that the photon blockade can be changed into the transparency in the circuit QED system when the probe field [21] becomes strong.

Motivated by recent studies on the nanomechanical quantum switch between different light wavelengths using optomechanical effects [22, 23] and progress in the circuit QED for the strong photon-qubit coupling, we will study the photon transmission in the circuit QED system which is coupled to a nanomechanical resonator, or an optomechanical system which is coupled to a superconducting qubit. Different from former studies [8-10], here we will focus on the effect of single mechanical resonator (or single artificial atom) on the 
EIT of the circuit QED systems (or optomechanical systems) and statistical properties of the output field in these systems. We will also study the amplification phenomena of the output field.

\section{THEORETICAL MODEL}

\section{A. Hamiltonian}

As schematically shown in Fig. 1, we study a capacitive coupling between a nanomechanical resonator and a supderconducting circuit QED system, which consists of a transmission line resonator (TRL) and a superconducting qubit. Such hybrid system can also be considered as an optomechanical system which is coupled to a superconducting qubit. We assume that a strong driving field with the frequency $\omega_{d}$ and a weak probe field with the frequency $\omega_{p}$ are applied to the TLR. The Hamiltonian of the whole system can be written as

$$
\begin{aligned}
H= & \hbar \omega_{0} c^{\dagger} c+\left[\frac{p^{2}}{2 m}+\frac{1}{2} m \omega_{m}^{2} q^{2}\right]+\hbar \frac{\omega_{q}}{2} \sigma_{z}-\chi c^{\dagger} c q \\
& +\hbar g\left(c^{\dagger} \sigma_{-}+c \sigma_{+}\right)+i \hbar\left(\Omega e^{-i \omega_{d} t} c^{\dagger}-\Omega^{*} e^{i \omega_{d} t} c\right) \\
& +i \hbar\left(\varepsilon e^{-i \omega_{p} t} c^{\dagger}-\varepsilon^{*} e^{i \omega_{p} t} c\right) .
\end{aligned}
$$

Here $c^{\dagger}$ and $c$ are the creation and annihilation operators of the cavity field in the TLR with the frequency $\omega_{0} . \sigma_{+}$and $\sigma_{-}$are the raising and lowering operators of the superconducting qubit with the transition frequency $\omega_{q}$, they are expressed $\sigma_{ \pm}=\left(\sigma_{x} \pm i \sigma_{y}\right) / 2$ by Pauli spin operators $\sigma_{x}$ and $\sigma_{y}$. The parameters $q$ and $p$ represent the position and momentum operators of the mechanical resonator with the vibration frequency $\omega_{m}$ and the mass $m$. The coupling strengths of the cavity field to the strong driving field and weak probe field are $\Omega$ and $\varepsilon$, respectively. We assume $|\Omega| \gg|\varepsilon|$. The coupling between the cavity field and the mechanical resonator is characterized via the coupling strength $\chi$, while $g$ denotes the interaction strength between the superconducting qubit and the cavity field.

In the rotating reference frame at the frequency $\omega_{d}$ of the driving field, the Hamiltonian in Eq. (1) becomes

$$
\begin{aligned}
H= & \hbar \Delta_{1} c^{\dagger} c+\left(\frac{p^{2}}{2 m}+\frac{1}{2} m \omega_{m}^{2} q^{2}\right)+\frac{\hbar}{2} \Delta_{2} \sigma_{z} \\
& +\hbar g\left(c^{\dagger} \sigma_{-}+c \sigma_{+}\right)-\chi c^{\dagger} c q+i \hbar\left(\Omega c^{\dagger}-\Omega^{*} c\right) \\
& +i \hbar\left[\varepsilon e^{-i \Delta t} c^{\dagger}-\varepsilon^{*} e^{i \Delta t} c\right]
\end{aligned}
$$

with the detunings $\Delta_{1}=\omega_{0}-\omega_{d}, \Delta_{2}=\omega_{q}-\omega_{d}$, and $\Delta=$ $\omega_{p}-\omega_{d}$.

\section{B. Langevin equations}

Based on the Hamiltonian in Eq. (2) and phenomenologically adding the noise and decay terms, the HeisenbergLangevin equations of the variables for the mechanical resonator, the cavity field, and the superconducting qubit can be given as

$$
\begin{aligned}
\dot{q}= & \frac{p}{m} \\
\dot{p}= & -m \omega_{m}^{2} q-\gamma_{m} p+\chi c^{\dagger} c+\xi(t), \\
\dot{c}= & -\left(\gamma_{c}+i \Delta_{1}\right) c+\frac{i \chi}{\hbar} c q-i g \sigma_{-} \\
& +\Omega+\varepsilon e^{-i \Delta t}+\sqrt{2 \gamma_{c}} c_{\mathrm{in}}(t), \\
\dot{\sigma}_{-}= & -\left(\gamma_{a}+i \Delta_{2}\right) \sigma_{-}+i g c \sigma_{z}+\sqrt{2 \gamma_{a}} d_{\mathrm{in}}(t) .
\end{aligned}
$$

Here $\gamma_{c}, \gamma_{m}$, and $\gamma_{a}$ are the decay rates of the cavity field, the mechanical resonator, and the superconducting qubit. Equations of motion for the variables $c^{\dagger}$ and $\sigma_{+}$can be given by taking the Hermitian conjugate of Eqs. (5) and (6). We do not write down the equation of motion for the atomic operator $\sigma_{z}$ which will be explained below. $c_{\text {in }}(t)$ and $d_{\text {in }}(t)$ are the input vacuum noises of the cavity field and the superconducting qubit, and their mean values are zeroes

$$
\begin{aligned}
& \left\langle c_{\text {in }}(t)\right\rangle=0, \\
& \left\langle d_{\text {in }}(t)\right\rangle=0
\end{aligned}
$$

where $\langle\cdot\rangle$ represents the average over the equilibrium state of the environment. We assume that the system works at low temperature, and thus the eigen-energies of the qubit and the cavity field are much higher than the thermal energy, which means that the thermal effects on the cavity field and the qubit are negligible. With this assumption, the vacuum noises $c_{\mathrm{in}}(t)$ and its Hermitian conjugate, $d_{\text {in }}(t)$ and its Hermitian conjugate, satisfy the following conditions

$$
\begin{aligned}
& \left\langle c_{\text {in }}(t) c_{\text {in }}^{\dagger}\left(t^{\prime}\right)\right\rangle=\delta\left(t-t^{\prime}\right), \\
& \left\langle d_{\text {in }}(t) d_{\text {in }}^{\dagger}\left(t^{\prime}\right)\right\rangle=\delta\left(t-t^{\prime}\right) .
\end{aligned}
$$

Since the frequency of the mechanical resonator is much smaller than the frequency of the cavity field and the transition frequency of the qubit, the thermal motion of the mechanical resonator should be taken into account. Thus we introduce the thermal Langevin force $\xi(t)$ with the zero mean value $\langle\xi(t)\rangle=0$ and satisfies the following temperature-dependent correlation function [2, 24]

$$
\left\langle\xi(t) \xi\left(t^{\prime}\right)\right\rangle=\frac{1}{2 \pi} \int \exp \left[-i \omega\left(t-t^{\prime}\right)\right] N(\omega) d \omega,
$$

with

$$
N(\omega)=\hbar \gamma_{m} m \omega\left[1+\operatorname{coth}\left(\frac{\hbar \omega}{2 k_{B} T}\right)\right]
$$

\section{STEADY-STATE SOLUTIONS AND PHOTON TRANSMISSION}

We are interested in the effect of the mechanical resonator on the photon transmission through the circuit QED system, or, equivalently, the effect of the superconducting qubit on the photon transmission through the optomechanical system. 


\section{A. Steady-state solutions}

Using Eqs. (3) 8 and also the mean field approximation, e.g., $\langle c q\rangle=\langle c\rangle\langle q\rangle$, the time evolutions of the expectation values for the operators $q, p, c$, and $\sigma_{-}$are given by

$$
\begin{aligned}
\frac{d\langle q\rangle}{d t}= & \frac{\langle p\rangle}{m}, \\
\frac{d\langle p\rangle}{d t}= & -m \omega_{m}^{2}\langle q\rangle-\gamma_{m}\langle p\rangle+\chi\left\langle c^{\dagger}\right\rangle\langle c\rangle, \\
\frac{d\langle c\rangle}{d t}= & -\left(\gamma_{c}+i \Delta_{1}-\frac{i \chi}{\hbar}\langle q\rangle\right)\langle c\rangle-i g\left\langle\sigma_{-}\right\rangle \\
& +\Omega+\varepsilon e^{-i \Delta t}, \\
\frac{d\left\langle\sigma_{-}\right\rangle}{d t}= & -\left(\gamma_{a}+i \Delta_{2}\right)\left\langle\sigma_{-}\right\rangle+i g\langle c\rangle\left\langle\sigma_{z}\right\rangle .
\end{aligned}
$$

The equations of motion for the average of the operators $c^{\dagger}$ and $\sigma_{+}$can be obtained by taking the average for the Hermitian conjugates of Eq. (5) and Eq. (6), respectively. The nonlinear equations in Eqs. (13 16) cannot be solved precisely since their steady-state solutions have infinite number of frequencies. To approximately obtain the steady-state solutions which are exact for the strong driving $\Omega$ and are correct to the lowest order in the weak probe $\varepsilon$, we make the following ansatz [25]

$$
\begin{aligned}
\langle c\rangle & =C_{0}+C_{+} e^{i \Delta t}+C_{-} e^{-i \Delta t} \\
\langle q\rangle & =Q_{0}+Q_{+} e^{i \Delta t}+Q_{-} e^{-i \Delta t} \\
\left\langle\sigma_{-}\right\rangle & =L_{0}+L_{+} e^{i \Delta t}+L_{-} e^{-i \Delta t} .
\end{aligned}
$$

Here $C_{ \pm}$are much smaller than $C_{0}$, and are of the same order of $\varepsilon$. Similarly, $Q_{ \pm}$and $L_{ \pm}$are much smaller than $Q_{0}$ and $L_{0}$. The parameters $Q_{ \pm}$and $L_{ \pm}$are of the same order of $\varepsilon . C_{0}$, $Q_{0}$, and $L_{0}$ are the steady-state solutions when $\varepsilon=0$.

By substituting Eqs. (17, 19) into Eqs. (13,16), and keeping the first-order terms of $Q_{ \pm}$and $L_{ \pm}$, we can give

$$
\begin{aligned}
Q_{0} & =\frac{\chi}{m \omega_{m}^{2}}\left|C_{0}\right|^{2} \\
Q_{ \pm} & =\frac{\chi}{m\left(\omega_{m}^{2} \pm i \gamma_{m} \Delta-\Delta^{2}\right)}\left(C_{0} C_{\mp}^{*}+C_{0}^{*} C_{ \pm}\right),
\end{aligned}
$$

by comparing the coefficients of the terms with the same frequency. Similarly, the expressions of $L_{0}, L_{+}$, and $L_{-}$can also be obtained as

$$
\begin{aligned}
L_{0} & =\frac{i g\left\langle\sigma_{z}\right\rangle_{s s} C_{0}}{\left(\gamma_{a}+i \Delta_{2}\right)}, \\
L_{ \pm} & =\frac{i g\left\langle\sigma_{z}\right\rangle_{s s} C_{ \pm}}{\left(\gamma_{a}+i \Delta_{2} \pm i \Delta\right)},
\end{aligned}
$$

where $\left\langle\sigma_{z}\right\rangle_{s s}$ is the steady-state value of the operator $\left\langle\sigma_{z}\right\rangle$ for the superconducting qubit.

Using Eqs. 2023), we can obtain the steady-state values

$$
C_{0}=\frac{\Omega}{\gamma_{c}+i \Delta_{3}-\left[g^{2}\left\langle\sigma_{z}\right\rangle_{s s} /\left(\gamma_{a}+i \Delta_{2}\right)\right]}
$$

and

$$
C_{-}=\frac{\varepsilon\left(\lambda_{1}-\lambda_{2}\right)}{A+2 i \Delta_{3} \lambda_{3}+\lambda_{2}^{+} \lambda_{2}-\lambda_{2}^{+} \lambda_{1}-\lambda_{1}^{+} \lambda_{2}} .
$$

The parameter $A$ in Eq. (25) is expressed as $A=\left(\lambda_{1}-\right.$ $\left.\lambda_{3}\right)\left(\lambda_{1}^{+}+\lambda_{3}\right)$, and other parameters are given as

$$
\begin{aligned}
\Delta_{3} & =\Delta_{1}-\frac{\chi^{2}}{m \hbar \omega_{m}^{2}}\left|C_{0}\right|^{2}, \\
\lambda_{1} & \equiv \lambda_{1}(\Delta)=\gamma_{c}-i \Delta_{3}-i \Delta+\frac{i \chi^{2}\left|C_{0}\right|^{2}}{M(\Delta)}, \\
\lambda_{2} & \equiv \lambda_{2}(\Delta)=\frac{g^{2}\left\langle\sigma_{z}\right\rangle_{s s}}{\left(\gamma_{a}-i \Delta_{2}-i \Delta\right)}, \\
\lambda_{3} & \equiv \lambda_{3}(\Delta)=\frac{i \chi^{2}\left|C_{0}\right|^{2}}{M(\Delta)} .
\end{aligned}
$$

with $M(\Delta)=m \hbar\left(\omega_{m}^{2}-i \gamma_{m} \Delta-\Delta^{2}\right)$. The parameters $\lambda_{1}^{+}$ and $\lambda_{2}^{+}$in Eq. (25) are defined as

$$
\begin{aligned}
\lambda_{1}^{+} \equiv \lambda_{1}^{+}(\Delta) & =\left[\lambda_{1}(-\Delta)\right]^{*}, \\
\lambda_{2}^{+} \equiv \lambda_{2}^{+}(\Delta) & =\left[\lambda_{2}(-\Delta)\right]^{*} .
\end{aligned}
$$

Here we are not interested in four-wave mixing with the frequency $\left(\omega_{p}-2 \omega_{d}\right)$, thus the expression of $C_{+}$is not written out.

\section{B. Output field and the response of the whole system to the probe field}

The response of the system to all frequencies can be detected by the output field, which can be given via the inputoutput theory [26, 27],

$$
c_{\text {in }}(t)+c_{\text {out }}(t)=\sqrt{2 \gamma_{c}} c-\frac{1}{\sqrt{2 \gamma_{c}}}\left(\Omega+\varepsilon e^{-i \Delta t}\right) .
$$

Using Eq. (17) and considering the zero mean value for the vacuum input field, i.e., $\left\langle c_{\mathrm{in}}(t)\right\rangle=0$, we can express the mean value of the output field via Eq. (32) as

$$
\begin{aligned}
\sqrt{2 \gamma_{c}}\left\langle c_{\text {out }}(t)\right\rangle & =\left(2 \gamma_{c} C_{0}-\Omega\right)+\left(2 \gamma_{c} \frac{C_{-}}{\varepsilon}-1\right) \varepsilon e^{-i \Delta t} \\
& +2 \gamma_{c}\left(\frac{C_{+}}{\varepsilon^{*}}\right) \varepsilon^{*} e^{i \Delta t} .
\end{aligned}
$$

We can find that the second term in the right side of Eq. (33) corresponds to the response of the whole system to the probe field with the frequency $\omega_{p}$. Thus the real and imaginary parts of the amplitude of this term describe the absorption and dispersion of the whole system to the probe field. Since a constant does not change the lineshape of a signal, we can define the amplitude of the rescaled output field corresponding to the probe field $\varepsilon e^{-i \omega_{p} t}$ as

$$
\varepsilon_{\text {out }}=\frac{2}{\varepsilon} \gamma_{c} C_{-} .
$$



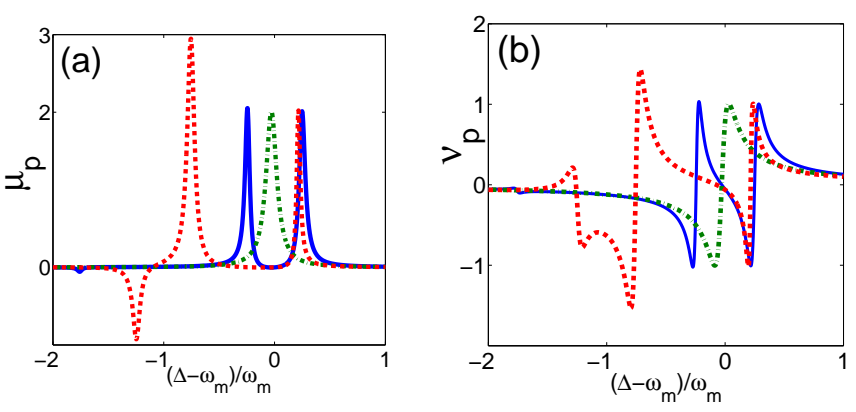

FIG. 2: (Color online) Real and imaginary parts $\mu_{p}$ and $\nu_{p}$ of the rescaled output field $\varepsilon_{\text {out }}$ given in Eq. (34) versus the normalized detuning parameter $\left(\Delta-\omega_{m}\right) / \omega_{m}$ are plotted in (a) and (b) with different parameters: (i) $g=0$ and $\chi / 2 \pi=2.8 \times 10^{-14} \mathrm{~J} / \mathrm{m}$ (blue solid curve); (ii) $g / 2 \pi=41.7 \mathrm{MHz}$ and $\chi=0$ (green dash-dotted curve); and (iii) $g / 2 \pi=41.7 \mathrm{MHz}$ and $\chi / 2 \pi=2.8 \times 10^{-14} \mathrm{~J} / \mathrm{m}$ (red dashed curve). The frequency and damping rate of the cavity are $\omega_{0} / 2 \pi=5 \mathrm{GHz}$ and $\gamma_{c} / 2 \pi=0.5 \mathrm{MHz}$. The transition frequency and decoherence rate of the superconducting qubit are $\omega_{q} / 2 \pi=4$ $\mathrm{GHz}$ and $\gamma_{a} / 2 \pi=1 \mathrm{MHz}$. The parameters of the mechanical resonator are assumed as $\omega_{m} / 2 \pi=8.5 \mathrm{MHz}, \gamma_{m} / 2 \pi=25 \mathrm{~Hz}$, and $m=2 \times 10^{-15} \mathrm{~kg}$. The frequency of the driving field is chosen to be $\omega_{d} / 2 \pi=4.99 \mathrm{GHz}$. The coupling strength between the cavity field and the driving field is designated as $\Omega / 2 \pi=3.1 \mathrm{MHz}$.

The real and imaginary parts of $\varepsilon_{\text {out }}$ are given as

$$
\begin{aligned}
& \mu_{p}=\frac{\gamma_{c}\left(C_{-}+C_{-}^{*}\right)}{\varepsilon}, \\
& \nu_{p}=\frac{\gamma_{c}\left(C_{-}-C_{-}^{*}\right)}{i \varepsilon} .
\end{aligned}
$$

These two quadratures of the output field can be measured by homodyne detections [27]. Because we are interested in the photon transmission in the large detuning between the cavity field and superconducting qubit, thus, without loss of generality, hereafter we assume that the qubit always keeps at the excited state, that is, the steady-state value $\left\langle\sigma_{z}\right\rangle_{s s}$ is assumed as $\left\langle\sigma_{z}\right\rangle_{s s}=1$.

\section{Numerical simulations: electromagnetically induced transparency and amplification}

We now numerically simulate $\mu_{p}$ and $\nu_{p}$ in Figs. (2, 4) with experimentally accessible parameters in optomechanical system [14] and also superconducting qubit [15, 16]. In Fig.2] we first study the case that the cavity field and the qubit have very large detuning $\omega_{0}-\omega_{q}$ such that $g^{2} /\left(\omega_{0}-\omega_{q}\right)$ is small, but there is no coupling between the cavity field and the mechanical resonator. In this case, we find that there is no EIT and amplification to the weak probe field (see green dash-dotted curve in Fig. 2]a)). Second, we study the case that there is no coupling between the cavity field and the superconducting qubit, but there is a strong coupling between the cavity field and mechanical resonator. We find that there is an analogue of the EIT (see blue solid curves of Figs. 2(a) and (b))
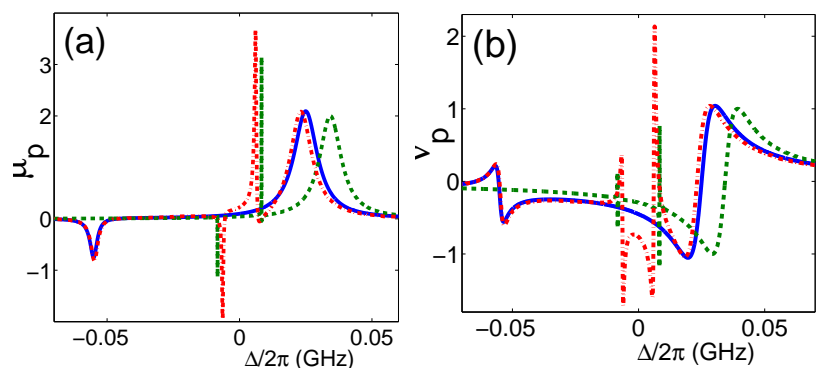

FIG. 3: (Color online) Real and imaginary parts $\mu_{p}$ and $\nu_{p}$ of the output field $\varepsilon_{\text {out }}$ given in Eq. (34) versus the detuing $\Delta=\omega_{p}-\omega_{d}$ are plotted in (a) and (b) with different parameters: (i) $g / 2 \pi=30$ MHz and $\chi=0$ (blue solid curve); (ii) $g=0$ and $\chi / 2 \pi=3.0 \times$ $10^{-13} \mathrm{~J} / \mathrm{m}$ (green dashed curve); and (iii) $g / 2 \pi=30 \mathrm{MHz}$ and $\chi / 2 \pi=3 \times 10^{-13} \mathrm{~J} / \mathrm{m}$ (red dash-dotted curve). The parameters of the cavity are assumed as $\omega_{0} / 2 \pi=5 \mathrm{GHz}$ and $\gamma_{c} / 2 \pi=5 \mathrm{MHz}$. The parameters of the superconducting qubit are taken as $\omega_{q} / 2 \pi=$ $4.9 \mathrm{GHz}$ and $\gamma_{a} / 2 \pi=2 \mathrm{MHz}$. The parameters of the mechanical resonator are designated as $\omega_{m} / 2 \pi=8.5 \mathrm{MHz}, \gamma_{m} / 2 \pi=25 \mathrm{~Hz}$, and $m=2 \times 10^{-15} \mathrm{~kg}$. The frequency of the driving field is assumed as $\omega_{d} / 2 \pi=4.965 \mathrm{GHz}$. The coupling strength between the driving field and the cavity field is $\Omega / 2 \pi=0.98 \mathrm{MHz}$.

as experimentally demonstrated in other optomechanical systems [5-7]. Moreover, we can also observe the amplification of the weak probe field (see small dip of the blue solid curve of Fig. 2(a)). Finally, we study the case that $g^{2} /\left(\omega_{0}-\omega_{q}\right)$ is small but the coupling between the cavity field and mechanical resonator is strong. We find that the coupling of the qubit to the cavity field modifies the absorption and dispersive curves of the optomechanical systems (see red dashed curves in Figs. 2(a) and (b)). Particularly, the qubit broadens the transparency windows [9] and also helps the optomechanical system to significantly amplify the probe field in some parameter regimes (see dip in red dashed curve in Fig.2(a)).

We further numerically study the case for both the bigger $g^{2} /\left(\omega_{0}-\omega_{q}\right)$ and the strong optomechanical coupling in Fig. 3. We find: i) with the increase of the optomechanical coupling strength $\chi$, the shapes of the absorption and dispersion curves become asymmetric when there is no coupling between the qubit and the cavity field, and also the weak probe field is amplified greatly (see green dashed curves in Figs.3 a) and (b)); ii) the bigger $g^{2} /\left(\omega_{0}-\omega_{q}\right)$ can result in the EIT [21] and amplification even without the optomechanical coupling, i.e., $\chi=0$ (see blue solid curves in Figs. 3 (a) and (b)); and iii) the strong optomechanical coupling $\chi$ can significantly modify the transparency windows and dispersive curves even when $g^{2} /\left(\omega_{0}-\omega_{q}\right)$ is bigger (see red dash-dotted curves in Figs. 3(a) and (b)).

In Fig. 4 we study the effects of the optomechanical coupling strength $\chi$ and the qubit-cavity coupling strength $g$ on the transparency windows by plotting the real part $\mu_{p}$ of the output field given in Eq. (34). We first fix other parameters and study how the transparency windows change with the coupling strength $g$ in Fig. 4(a). We find that $g$ affects not only the position and width of the transparency windows but also the 

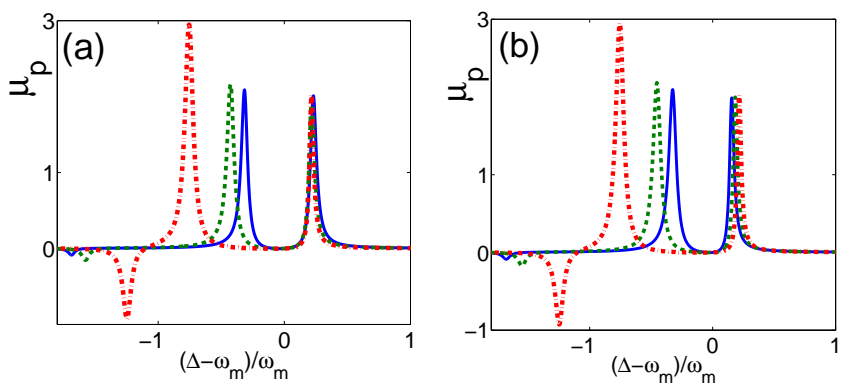

FIG. 4: (Color online) The real part $\mu_{p}$ of the output field given in Eq. (34) versus the normalized detuning parameter $\left(\Delta-\omega_{m}\right) / \omega_{m}$ is plotted in (a) with different system parameters: $g / 2 \pi=21.7 \mathrm{MHz}$ (blue solid curve), $g / 2 \pi=31.7 \mathrm{MHz}$ (green dashed curve), and $g / 2 \pi=41.7 \mathrm{MHz}$ (red dash-dotted curve), with that other parameters are the same as in Fig.2]a). Similarly, we also plot $\mu_{p}$ in (b) with different optomechanical coupling strengths: $\chi / 2 \pi=2 \times 10^{-14} \mathrm{~J} / \mathrm{m}$ (blue solid curve), $\chi / 2 \pi=2.4 \times 10^{-14} \mathrm{~J} / \mathrm{m}$ (green dashed curve), and $\chi / 2 \pi=2.8 \times 10^{-14} \mathrm{~J} / \mathrm{m}$ (red dash-dotted curve), with that other parameters are the same as in Fig. 2 a).

gain of the output field. In particular, we can find that the large coupling strength $g$ leads to a high gain of the output field. We then fix other parameters and study how the coupling strength $\chi$ affects the transparency windows in Fig. 4(b). We find that $\chi$ has similar effects on the transparency windows and gain of the output field as the coupling constant $g$. Thus, Fig. 4tells us that both increasing coupling constants $\chi$ and $g$ can efficiently amplify the weak input field in some parameter regimes.

\section{QUANTUM STATISTICAL PROPERTIES OF THE OUTPUT FIELD}

For the hybrid system studied here, one would like to know the statistical properties of the output field. To demonstrate this, let us now study the degrees of second-order coherence for the output field. In this case, there are vacuum input field and strong driving field, but the weak probe field is turned off.

\section{A. Small fluctuation approximation}

We assume that the whole system reaches the steady-state and there are small fluctuations near this steady-state. As discussed in subsection III.A, this steady-state can be obtained by the average of system operators given in Eqs. (13 [16) with $\varepsilon=0$. Similar to Eqs. (17, 19), we assume that the steadystate values of the position and momentum of the mechanical resonator are $Q_{0}$ and $P_{0}$, and the steady-state values of the cavity annihilation operator and the qubit ladder operator are $C_{0}$ and $L_{0}$. With these notations, the system operators $q, p$, $\sigma_{-}$, and $c$ can be expressed as

$$
\begin{aligned}
& q=Q_{0}+Q, \\
& p=P_{0}+P, \\
& \sigma_{-}=L_{0}+\sigma, \\
& c=C_{0}+C,
\end{aligned}
$$

where $Q, P, \sigma, C$ represent the fluctuation operators of $q, p$, $\sigma_{-}, c$ around the steady-state values $Q_{0}, P_{0}, L_{0}, C_{0}$. From Eqs. (3) 6, we can obtain $P_{0}=0$. Moreover, the steady-state values $Q_{0}, L_{0}$, and $C_{0}$ can be calculated by Eqs. (20), 22), and (24), respectively. It is also clear that the mean values of all small fluctuation operators $Q, P, \sigma$, and $C$ are zeroes.

We now assume $\varepsilon=0$ in Eqs. (3.6) and substitute Eqs. (36. 39) into Eqs. (3) 6), then we can linearize Eqs. (3) 6) up to first order of fluctuation operators, and obtain linear dynamical equations of four fluctuation operators as:

$$
\begin{aligned}
\dot{Q}(t)= & \frac{P(t)}{m} \\
\dot{P}(t)= & -m \omega_{m}^{2} Q(t)-\gamma_{m} P(t)+\chi C_{0}^{*} C(t) \\
& +\chi C_{0} C^{\dagger}(t)+\xi(t) \\
\dot{C}(t)= & -\left(\gamma_{c}+i \Delta_{1}-\frac{i \chi}{\hbar} Q_{0}\right) C(t)+\frac{i \chi C_{0}}{\hbar} Q(t) \\
& -i g \sigma(t)+\sqrt{2 \gamma_{c}} c_{\text {in }}(t) \\
\dot{\sigma}(t)= & -\left(\gamma_{a}+i \Delta_{2}\right) \sigma(t)+i g\left\langle\sigma_{z}\right\rangle_{s s} C(t) \\
& +\sqrt{2 \gamma_{a}} d_{\text {in }}(t)
\end{aligned}
$$

Here, the operator $C^{\dagger}(t)$ and the steady-state value $C_{0}^{*}$ satisfy $c^{\dagger}=C_{0}^{*}+C^{\dagger}(t)$. The equation of the operator $C^{\dagger}(t)$ can be given by taking the Hermitian conjugate of Eq. (42). Using the Fourier transform with the definition

$$
f(t)=\frac{1}{2 \pi} \int_{-\infty}^{\infty} f(\omega) \exp (-i \omega t) d \omega
$$

Eqs. (40,43) can be given in the frequency domain as

$$
\begin{aligned}
-i \omega Q(\omega)= & \frac{P(\omega)}{m} \\
-i \omega P(\omega)= & -m \omega_{m}^{2} Q(\omega)-\gamma_{m} P(\omega)+\chi C_{0}^{*} C(\omega) \\
& +\chi C_{0} C^{\dagger}(\omega)+\xi(\omega) \\
-i \omega C(\omega)= & -\left(\gamma_{c}+i \Delta_{1}-\frac{i \chi}{\hbar} Q_{0}\right) C(\omega)-i g \sigma(\omega) \\
& +\frac{i \chi C_{0}}{\hbar} Q(\omega)+\sqrt{2 \gamma_{c}} c_{\mathrm{in}}(\omega) \\
-i \omega \sigma(\omega)= & -\left(\gamma_{a}+i \Delta_{2}\right) \sigma(\omega)+i g\left\langle\sigma_{z}\right\rangle_{s s} C(\omega) \\
& +\sqrt{2 \gamma_{a}} d_{\mathrm{in}}(\omega) .
\end{aligned}
$$


Let us now define the frequency dependent parameters

$$
\begin{aligned}
E(\omega)= & \left(\Lambda_{1}-\Lambda_{3}\right)\left(\Lambda_{1}^{+}-\Lambda_{3}\right)+2 i \Delta_{3} \Lambda_{3} \\
& +\Lambda_{2}^{+} \Lambda_{2}-\Lambda_{2}^{+} \Lambda_{1}-\Lambda_{1}^{+} \Lambda_{2}, \\
F(\omega)= & \lambda_{1}-\lambda_{2}, \\
R(\omega)= & \frac{1}{m \hbar\left(\omega_{m}^{2}-i \gamma_{m} \omega-\omega^{2}\right)}, \\
S(\omega)= & \frac{1}{\left(\gamma_{a}-i \Delta_{2}-i \omega\right)}, \\
T(\omega)= & \frac{1}{\left(\gamma_{a}+i \Delta_{2}-i \omega\right)} .
\end{aligned}
$$

Here the parameter $\Delta_{3}$ is given in Eq. (26). The parameters $\Lambda_{1}, \Lambda_{2}$, and $\Lambda_{3}$ can be obtained through Eqs. (27,29) by replacing $\Delta$ with $\omega$, respectively. Using Eqs. (45,48) and also the Fourier transform of the equation of $C^{\dagger}(t)$, we can obtain the solutions of the fluctuation operators in the frequency domain. Specifically, we have

$$
\begin{aligned}
C(\omega)= & C_{1}(\omega) c_{\mathrm{in}}(\omega)+C_{2}(\omega) c_{\mathrm{in}}^{\dagger}(\omega)+C_{3}(\omega) d_{\mathrm{in}}(\omega) \\
& +C_{4}(\omega) d_{\mathrm{in}}^{\dagger}(\omega)+C_{5}(\omega) \xi(\omega)
\end{aligned}
$$

where the parameters $C_{i}(\omega)$, with $i=1,2,3,4,5$, are given by

$$
\begin{aligned}
& C_{1}(\omega)=\frac{\sqrt{2 \gamma_{c}} F(\omega)}{E(\omega)}, \\
& C_{2}(\omega)=\frac{i \chi^{2} C_{0}^{2} \sqrt{2 \gamma_{c}} R(\omega)}{E(\omega)}, \\
& C_{3}(\omega)=-\frac{i g \sqrt{2 \gamma_{a}} F(\omega) T(\omega)}{E(\omega)}, \\
& C_{4}(\omega)=-\frac{\chi^{2} C_{0}^{2} g \sqrt{2 \gamma_{a}} R(\omega) S(\omega)}{E(\omega)}, \\
& C_{5}(\omega)=\frac{\chi^{3}\left|C_{0}\right|^{2} C_{0} R^{2}(\omega)+i \chi C_{0} F(\omega) R(\omega)}{E(\omega)} .
\end{aligned}
$$

\section{B. Degrees of second-order coherence for output fields}

By setting $\varepsilon=0$ in Eq. (32) and also using Fourier transform, we can obtain the input-output relation in the frequency domain as

$$
2 \gamma_{c} c(\omega)=\sqrt{2 \gamma_{c}}\left[c_{\text {out }}(\omega)+c_{\text {in }}(\omega)\right]+2 \pi \Omega \delta(\omega) .
$$

Thus, using Eq. (39) and Eq. (54), the output field in Eq. (56) can be given as

$$
c_{\text {out }}(\omega)=2 \pi B_{0} \delta(\omega)+G(\omega)
$$

with

$$
B_{0}=\sqrt{2 \gamma_{c}} C_{0}-\frac{\Omega}{\sqrt{2 \gamma_{c}}}
$$

and

$$
\begin{aligned}
G(\omega)= & B_{1}(\omega) c_{\mathrm{in}}(\omega)+B_{2}(\omega) c_{\mathrm{in}}^{\dagger}(\omega)+B_{3}(\omega) d_{\mathrm{in}}(\omega) \\
& +B_{4}(\omega) d_{\mathrm{in}}^{\dagger}(\omega)+B_{5}(\omega) \xi(\omega) .
\end{aligned}
$$

The parameters in Eq. (59) are

$$
\begin{aligned}
& B_{1}(\omega)=\sqrt{2 \gamma_{c}} C_{1}(\omega)-1, \\
& B_{2}(\omega)=\sqrt{2 \gamma_{c}} C_{2}(\omega), \\
& B_{3}(\omega)=\sqrt{2 \gamma_{c}} C_{3}(\omega), \\
& B_{4}(\omega)=\sqrt{2 \gamma_{c}} C_{4}(\omega), \\
& B_{5}(\omega)=\sqrt{2 \gamma_{c}} C_{5}(\omega) .
\end{aligned}
$$

To demonstrate the statistical properties of the output fields, let us now calculate the degrees of second-order coherence, which is defined as

$$
g^{(2)}(\tau)=\frac{\left\langle c_{\text {out }}^{\dagger}(t) c_{\text {out }}^{\dagger}\left(t^{\prime}\right) c_{\text {out }}\left(t^{\prime}\right) c_{\text {out }}(t)\right\rangle}{\left\langle c_{\text {out }}^{\dagger}(t) c_{\text {out }}(t)\right\rangle\left\langle c_{\text {out }}^{\dagger}\left(t^{\prime}\right) c_{\text {out }}\left(t^{\prime}\right)\right\rangle}
$$

with $t^{\prime}=t+\tau$. Using Eqs. [57,[59), we can obtain $g^{(2)}(\tau)$ with simple calculations as

$$
\begin{aligned}
g^{(2)}(\tau)= & \frac{2\left|B_{0}\right|^{2}\left\langle G^{\dagger}(t) G(t)\right\rangle+2 \Re\left[B_{0}^{* 2}\left\langle G\left(t^{\prime}\right) G(t)\right\rangle\right]}{\left(\left|B_{0}\right|^{2}+\left\langle G^{\dagger}(t) G(t)\right\rangle\right)^{2}} \\
& +\frac{\left|B_{0}\right|^{4}+2\left|B_{0}\right|^{2} \Re\left[\left\langle G^{\dagger}\left(t^{\prime}\right) G(t)\right\rangle\right]}{\left(\left|B_{0}\right|^{2}+\left\langle G^{\dagger}(t) G(t)\right\rangle\right)^{2}} \\
& +\frac{\left\langle G^{\dagger}(t) G^{\dagger}\left(t^{\prime}\right) G\left(t^{\prime}\right) G(t)\right\rangle}{\left(\left|B_{0}\right|^{2}+\left\langle G^{\dagger}(t) G(t)\right\rangle\right)^{2}},
\end{aligned}
$$

where $\Re[\cdot]$ represents the real part of the complex number. Note that the correlation function $R(\tau)=$ $\left\langle G^{\dagger}(t) G^{\dagger}\left(t^{\prime}\right) G\left(t^{\prime}\right) G(t)\right\rangle$ can be calculated via the Fourier transform

$$
\begin{aligned}
& R(\tau)=\left\langle G^{\dagger}(t) G^{\dagger}\left(t^{\prime}\right) G\left(t^{\prime}\right) G(t)\right\rangle \\
& =\alpha \iiint \int_{-\infty}^{+\infty}\left\langle G^{\dagger}\left(\omega_{1}\right) G^{\dagger}\left(\omega_{2}\right) G\left(\omega_{3}\right) G\left(\omega_{4}\right)\right\rangle \\
& \times e^{-i \omega_{1} t} e^{-i \omega_{2} t^{\prime}} e^{-i \omega_{3} t^{\prime}} e^{-i \omega_{4} t} d \omega_{1} d \omega_{2} d \omega_{3} d \omega_{4},
\end{aligned}
$$

with the normalization coefficient $\alpha=1 /(2 \pi)^{4}$. From Eqs. (9-11) and also the definition of Fourier transform given in Eq. (44), we can obtain the non-zero correlation functions of the input noise in the frequency domain as

$$
\begin{aligned}
\left\langle c_{\mathrm{in}}\left(\omega^{\prime}\right) c_{\mathrm{in}}^{\dagger}(\omega)\right\rangle & =2 \pi \delta\left(\omega+\omega^{\prime}\right), \\
\left\langle d_{\mathrm{in}}\left(\omega^{\prime}\right) d_{\mathrm{in}}^{\dagger}(\omega)\right\rangle & =2 \pi \delta\left(\omega+\omega^{\prime}\right), \\
\left\langle\xi(\omega) \xi\left(\omega^{\prime}\right)\right\rangle & =2 \pi N(\omega) \delta\left(\omega+\omega^{\prime}\right),
\end{aligned}
$$

where $N(\omega)$ is defined in Eq. (12). Since the stochastic force $\xi$ and vacuum inputs obey Gaussian distributions, we can calculate higher-order correlation functions using second-order correlation functions [28]. That is, Eq. (66) becomes 


$$
\begin{aligned}
4 \pi^{2} R(\tau) & =4 \pi^{2}\left\langle G^{\dagger}(t) G^{\dagger}\left(t^{\prime}\right) G\left(t^{\prime}\right) G(t)\right\rangle \\
& =\iint_{-\infty}^{+\infty} Y_{12}^{*}\left(\omega_{1}\right) Y_{12}\left(\omega_{2}\right) e^{i\left(\omega_{1}-\omega_{2}\right) \tau} d \omega_{1} d \omega_{2} \\
& +\iint_{-\infty}^{+\infty} Y_{13}\left(\omega_{1}\right) Y_{13}\left(\omega_{2}\right) e^{-i\left(\omega_{1}-\omega_{2}\right) \tau} d \omega_{1} d \omega_{2} \\
& +\iint_{-\infty}^{+\infty} Y_{14}\left(\omega_{1}\right) Y_{14}\left(\omega_{2}\right) d \omega_{1} d \omega_{2},
\end{aligned}
$$

where the parameters in Eq. (70) are given as

$$
\begin{aligned}
Y_{12}(\omega)= & N(\omega) B_{5}(-\omega) B_{5}(\omega)+B_{2}(-\omega) B_{1}(\omega) \\
& +B_{4}(-\omega) B_{3}(\omega), \\
Y_{13}(\omega)= & N(-\omega)\left|B_{5}(\omega)\right|^{2}+\left|B_{2}(\omega)\right|^{2}+\left|B_{4}(\omega)\right|^{2},(72) \\
Y_{14}(\omega)= & N(-\omega)\left|B_{5}(\omega)\right|^{2}+\left|B_{2}(\omega)\right|^{2}+\left|B_{4}(\omega)\right|^{2} .(73)
\end{aligned}
$$

Let us define

$$
\begin{aligned}
& y_{14}=\frac{1}{2 \pi} \int_{-\infty}^{+\infty} Y_{14}(\omega) d \omega \\
& y_{13}=\frac{1}{2 \pi} \int_{-\infty}^{+\infty} Y_{13}(\omega) e^{i \omega \tau} d \omega \\
& y_{12}=\frac{1}{2 \pi} \int_{-\infty}^{+\infty} Y_{12}(\omega) e^{-i \omega \tau} d \omega
\end{aligned}
$$

then the degrees of second-order coherence can be further simplified to

$$
\begin{aligned}
g^{(2)}(\tau)= & \frac{\left|B_{0}\right|^{4}+2\left|B_{0}\right|^{2} y_{14}+2 \Re\left[B_{0}^{* 2} y_{12}\right]+2\left|B_{0}\right|^{2} \Re\left[y_{13}\right]}{\left(\left|B_{0}\right|^{2}+y_{14}\right)^{2}} \\
& +\frac{y_{14}^{2}+\left|y_{13}\right|^{2}+\left|y_{12}\right|^{2}}{\left(\left|B_{0}\right|^{2}+y_{14}\right)^{2}}
\end{aligned}
$$

\section{Numerical simulations: Degrees of second-order coherence}

Let us numerically calculate the degrees of second-order coherence $g^{(2)}(\tau)$ as shown in Eq. (77) with different coupling constants $g$ and $\chi$ in Fig. 5 at the zero temperature. We find that the statistical properties of the output field strongly depend on the parameters. For example, the output field exhibits nonclassical properties in the case that $\chi$ is non-zero (see, the blue solid curve in Fig. 5 with $\chi / 2 \pi=2.8 \times 10^{-14} \mathrm{~J} / \mathrm{m}$, and $g=0$ ). When $\chi=0$ and the coupling constant $g / 2 \pi=41.7$ $\mathrm{MHz}$, it is hard to observe nonclassical properties of the output field. However, further calculations of $g^{(2)}(\tau)$ show that the output field gradually exhibits non-classical properties with the increase of the coupling constant $g$ when $\chi=0$. This can be well understood, because the large coupling strength $g$ results in a large effective photon-photon interaction inside the cavity, which leads to nonclassical properties of the cavity field as those with the giant Kerr effects. Finally, combining the effects of two non-zero coupling constants $\chi$ and $g$,

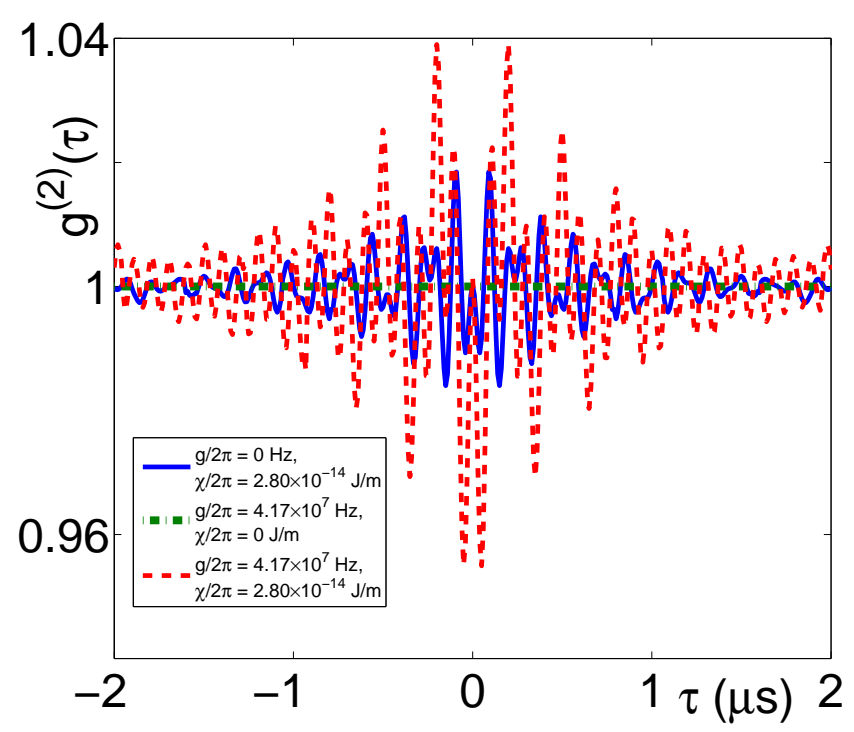

FIG. 5: (Color online) Degrees of second-order coherence $g^{(2)}(\tau)$ are plotted as the function of the time interval $\tau$ with different coupling constants: (i) $g=0$ and $\chi / 2 \pi=2.8 \times 10^{-14} \mathrm{~J} / \mathrm{m}$ (blue solid curve); (ii) $g / 2 \pi=41.7 \mathrm{MHz}$ and $\chi=0$ (green dash-dotted curve); and (iii) $g / 2 \pi=41.7 \mathrm{MHz}$ and $\chi / 2 \pi=2.8 \times 10^{-14} \mathrm{~J} / \mathrm{m}$ (red dashed curve). Here the parameters of the cavity are taken as $\omega_{0} / 2 \pi=5 \mathrm{GHz}$ and $\gamma_{c} / 2 \pi=0.5 \mathrm{MHz}$. The parameters of the superconducting qubit are designated as $\omega_{q} / 2 \pi=4 \mathrm{GHz}, \gamma_{a} / 2 \pi=1$ MHz. The parameters of the mechanical resonator are taken as $\omega_{m} / 2 \pi=8.5 \mathrm{MHz}, \gamma_{m} / 2 \pi=25 \mathrm{~Hz}$, and $m=2 \times 10^{-15} \mathrm{~kg}$. The frequency of the driving field is $\omega_{d} / 2 \pi=4.99 \mathrm{GHz}$, and the coupling strength between the driving field and the cavity field is $\Omega / 2 \pi=3.1 \mathrm{MHz}$.

we observe that the coupling between the cavity field and the superconducting qubit can enhance nonclassical properties of the output field.

To study the effects of the driving field on the statistical properties of the output field, we take all the parameters which are the same as those in Fig. 5 besides the parameter $\Omega$ and plot $g^{(2)}(\tau)$ as the function of the time interval $\tau$ in Fig. 6 with $\Omega / 2 \pi=0.22 \mathrm{MHz}$. Figure 6 shows that the weak driving field enhances the nonclassical properties of the output field. We also numerically simulate the thermal effects for different environmental temperature $T$ of the mechanical resonator and find that the non-classicality decreases with the increase of the temperature $T$.

\section{CONCLUSIONS AND REMARKS}

Our study here can be classified into two scenarios. One is a hybrid system formed by a circuit QED system and a mechanical resonator. Another equivalent one is the hybrid system consisting of an optomechanical system and a superconducting qubit. We mainly focus on the photon transmission in the case of the large detuning between the superconducting qubit and the cavity field. 


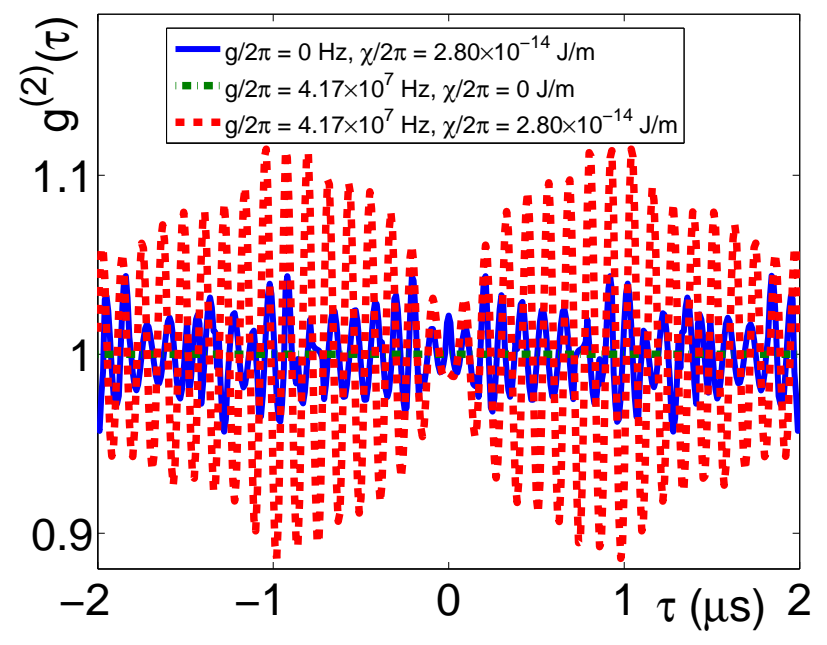

FIG. 6: Color online) Degrees of second-order coherence $g^{(2)}(\tau)$ is plotted as a function of time interval $\tau$ at the zero temperature with different coupling constants: (i) $g=0$ and $\chi / 2 \pi=2.8 \times 10^{-14} \mathrm{~J} / \mathrm{m}$ (blue solid curve); (ii) $g / 2 \pi=41.7 \mathrm{MHz}$ and $\chi=0$ (green dashdotted curve); and (iii) $g / 2 \pi=41.7 \mathrm{MHz}$ and $\chi / 2 \pi=2.8 \times 10^{-14}$ $\mathrm{J} / \mathrm{m}$ (red dashed curve). Here the parameters of the cavity are taken as $\omega_{0} / 2 \pi=5 \mathrm{GHz}$ and $\gamma_{c} / 2 \pi=0.5 \mathrm{MHz}$. The parameters of the superconducting qubit are designated as $\omega_{q} / 2 \pi=4 \mathrm{GHz}, \gamma_{a} / 2 \pi=$ $1 \mathrm{MHz}$. The parameters of the mechanical resonator are taken as $\omega_{m} / 2 \pi=8.5 \mathrm{MHz}, \gamma_{m} / 2 \pi=25 \mathrm{~Hz}$, and $m=2 \times 10^{-15} \mathrm{~kg}$. The frequency of the driving field is $\omega_{d} / 2 \pi=4.99 \mathrm{GHz}$, and the coupling strength between the driving field and the cavity field is $\Omega / 2 \pi=0.22 \mathrm{MHz}$.

In case one, we first find out the parameters, in the circuit QED system without the coupling to the mechanical resonator, that the EIT can be observed as in Ref. [21] in the large detuning condition. We find that these parameters should satisfy the strong coupling condition in the circuit QED system and $g^{2} /\left(\omega_{0}-\omega_{q}\right)$ should be big enough. We also find that the weak probe field can be amplified in the output in such a condition, while the negligibly small $g^{2} /\left(\omega_{0}-\omega_{q}\right)$ results in neither the transparency nor the amplification [21]. When the mechanical resonator is coupled to the circuit QED system with the big $g^{2} /\left(\omega_{0}-\omega_{q}\right)$, we find that the weak coupling between the mechanical resonator and cavity field can only slightly changes the shape of the transparency windows of the circuit QED system, and also slightly enhance the amplifica- tion of the weak probe field. However, when the optomechanical coupling becomes strong, the EIT and the amplification in the circuit QED are strongly distorted by the mechanical resonator.

In case two, we first find out experimentally accessible parameters for the analogue of the EIT and amplification of the weak probe field in optomechamical system without the coupling to the superconducting qubit. We then study the case that the superconducting qubit is coupled to the optomechanical system. In this case, even with a weak coupling between the qubit and the optomechanical system (i.e., with small but not negligible $\left.g^{2} /\left(\omega_{0}-\omega_{q}\right)\right)$, the qubit can broaden the transparency windows, and also help the optomechanical system to amplify the weak probe field.

We further explore the statistical properties of the output field in different parameter regimes. We find that both (i) the strong couplings between the cavity field and the mechanical resonator and (ii) the big $g^{2} /\left(\omega_{0}-\omega_{q}\right)$ can result in nonclassical output field with the weak driving field. We also find that both the high environmental temperature of the mechanical resonator and strong driving field can decrease nonclassical properties of the output field. Thus, the statistical properties of the output field strongly depend on the properties of each elements and the coupling strengths between different elements in hybrid system.

Because the superconducting qubit is easier to be controlled through external parameters and can also be coupled strongly to the cavity field, thus in contrast to our former study [8] in the optomechanical system with an uncontrollable atomic ensemble, we find that the properties of the photon transmission in the hybrid system studied here can be controlled by the tunable superconducting qubit. Our study provides a strategy for quantum switch [11] using a mechanical resonator (or a superconducting qubit) in an one-dimensional chain of the coupled circuit QED systems (or the optomechanical systems). Finally, we should also emphasize again that this study is only specified to the large detuning between the superconducting qubit and the cavity field, and the study for the resonant coupling is still under way.

\section{ACKNOWLEDGEMENT}

Y. X. Liu is supported by the National Natural Science Foundation of China under Nos. 10975080 and 61025022. J. Zhang is supported by the National Natural Science Foundation of China under Nos. 61174084 and 61134008.
[1] T. J. Kippenberg and K. J. Vahala, Science 321, 1172 (2008), and the references therein.

[2] G. S. Agarwal and S. M. Huang, Phys. Rev. A 81, 041803(R) (2010); S. M. Huang and G. S. Agarwal, Phys. Rev. A 83, 043826 (2011).

[3] C. L. Garrido Alzar, M. A. G. Martinez, and P. Nussenzveiga, Am. J. Phys. 70, 37 (2002).
[4] P. Tassin, L. Zhang, Th. Koschny, E. N. Economou, and C. M. Soukoulis, Phys. Rev. Lett. 102, 053901 (2009).

[5] S. Weis, R. Riviere, S. Deleglise, E. Gavartin, O. Arcizet, A. Schliesser, T. J. Kippenberg, Science 330, 1520 (2010).

[6] J. D. Teufel, D. Li, M. S. Allman, K. Cicak, A. J. Sirois, J. D. Whittaker, and R. W. Simmonds, Nature 471, 204 (2011).

[7] A. H. Safavi-Naeini, T. P. Mayer Alegre, J. Chan, M. Eichen- 
field, M. Winger, Q. Lin, J. T. Hill, D. E. Chang, and O. Painter, Nature 472, 69 (2011).

[8] H. Ian, Z. R. Gong, Yu-xi Liu, C. P. Sun, and F. Nori, Phys. Rev. A 78, 013824 (2008).

[9] Y. Han, J. Cheng, and L. Zhou, J. Phys. B: At. Mol. Opt. Phys. 44, 165505 (2011).

[10] Y. Chang, T. Shi, Yu-xi Liu, C. P. Sun, and F. Nori, Phys. Rev. A 83, 063826 (2011).

[11] C. P. Sun. L. F. Wei, Yu-xi Liu, and F. Nori, Phys. Rev. A 73, 022318 (2006).

[12] J. D. Teufel, T. Donner, D. Li, J. W. Harlow, M. S. Allman, K. Cicak, A. J. Sirois, J. D. Whittaker, K. W. Lehnert, and R. W. Simmonds, Nature 475, 359 (2011)

[13] C. A. Regal, J. D. Teufel, and K. W. Lehnert, Nature Phys. 4, 555 (2008).

[14] J. B. Hertzberg, T. Rocheleau, T. Ndukum, M. Savva, A. A. Clerk, and K. C. Schwab, Nature Phys. 6, 213 (2010); T. Rocheleau, T. Ndukum, C. Macklin, J. B. Hertzberg, A. A. Clerk, and K. C. Schwab, Nature 463, 72 (2010).

[15] R. J. Schoelkopf and S. M. Girvin, Nature 451, 664 (2008).

[16] J. Q. You and F. Nori, Phys. Today 58, 42 (2005); Nature 474, 589 (2011).

[17] H. Ian, Yu-xi Liu, and F. Nori, Phys. Rev. A 81, 063823 (2010).

[18] A. Imamoğlu, H. Schmidt, G. Woods, and M. Deutsch, Phys.
Rev. Lett. 79, 1467 (1997).

[19] C. Lang, D. Bozyigit, C. Eichler, L. Steffen, J. M. Fink, A. A. Abdumalikov, M. Baur, S. Filipp, M. P. da Silva, A. Blais, and A. Wallraff, Phys. Rev. Lett. 106, 243601 (2011).

[20] A. J. Hoffman, S. J. Srinivasan, S. Schmidt, L. Spietz, J. Aumentado, H. E. Türeci, and A. A. Houck, Phys. Rev. Lett. 107, 053602 (2011).

[21] Yu-xi Liu, X. W. Xu, A. Miranowicz, and F. Nori, arXiv:1203.6419

[22] L. Tian, Phys. Rev. Lett. 108, 153604 (2012).

[23] Y.-D. Wang and A. A. Clerk, Phys. Rev. Lett. 108, 153603 (2012).

[24] V. Giovannetti and D. Vitali, Phys. Rev. A 63, 023812 (2001).

[25] R. W. Boyd, Nonlinear Optics (Academic Press, New York, 2010).

[26] D. F. Walls and G. J. Milburn, Quantum Optics (Springer, Berlin, 1995).

[27] M. Orszag, Quantum Optics Including Noise Reduction, Trapped Ions, Quantum Trajectories, and Decoherence (Springer, Berlin, 2000).

[28] K. Borkje, A. Nunnenkamp, and S. M. Girvin, Phys. Rev. Lett. 107, 123601 (2011). 